\title{
Blue light cystoscopy with hexylaminolevulinate: Our 7 years experience
}

\author{
Vito Lacetera ${ }^{1}$, Ubaldo Cantoro ${ }^{1}$, Lorenzo Montesi ${ }^{1}$, Daniele Cantoro ${ }^{2}$, Bernardo Cervelli ${ }^{1}$, \\ Antonio Cicetti ${ }^{1}$, Giuliana Gabrielloni ${ }^{1}$, Domenico Milella ${ }^{1}$, Michele Montesi ${ }^{1}$, Roberto Morcellini ${ }^{1}$, \\ Gianni Parri ${ }^{1}$, Emilio Recanatini ${ }^{1}$, Valerio Beatrici ${ }^{1}$ \\ ${ }^{1}$ Azienda Ospedaliera Ospedali Riuniti Marche Nord, Pesaro-Fano, Italy; \\ ${ }^{2}$ Ospedale di Ascoli Piceno, Ascoli Piceno, Italy.
}

\begin{abstract}
Summary Aim: The objective of the present study is to evaluate the diagnostic accuracy of hexylaminolevulinate (HAL) blue light cystoscopy compared with standard white light cystoscopy (WLC) in daily practice. Materials and methods: An observational, comparative, controlled (within patient) study was carried out at our Center. 61 consecutive patients with suspected or confirmed bladder cancer were recruited for the study from January 2008 until January 2015. Patients with suspected bladder cancer (positive cytology with negative WLC) or history of previous high-grade NMIBC or CIS were included in the study. Biopsies/resection of each positive lesion/suspicious areas were always taken after the bladder was inspected under WLC and BLC. Diagnoses of bladder tumor or CIS were considered as positive results, and the presence of normal urothelium in the biopsy specimen as negative result. Results: 61 BLC were performed. 15/61 (24.5\%) with suspected initial diagnosis of NMIBC and 46/61 (75.5\%) with a history of high-risk non-muscle invasive bladder cancer (NMIBC). We performed a total of 173 biopsies/TURBT of suspicious areas: 129 positive only to the BLC and 44 both positive to WLC and BLC. 84/173 biopsies/TURBT were positive for cancer. All 84 NMIBC were positive to the BLC, while $35 / 84$ were positive to the WLC with a sensitivity of BLC and WLC respectively of $100 \%$ and $41.7 \%$. Sensitivity of WLC for highgrade NMIBC and CIS was $34.1 \%$ and $39 \%$ respectively while sensitivity of BLC for high-grade NMIBC and CIS was $100 \%$. The specificity of the WLC was $79.9 \%$ compared to $48.5 \%$ of the BLC. The positive predictive value of BLC and WLC were respectively $48 \%$ (95\% CI: $0.447-0.523)$ and $79 \%$ (95\% CI: 0.856-0.734).

Conclusions: Our data confirm those reported in the literature: BLC increases the detection rate of NMIBC particularly in high risk patients (history of CIS or high grade). BLC is a powerful diagnostic tool in the diagnosis of bladder cancer if malignancy is suspected (positive urine cytology) and if conventional WLC is negative.
\end{abstract}

KEY WORDS: Blue light cystoscopy; Hexaminolevulinate.

Submitted 7 February 2016; Accepted 3 June 2016

\section{INTRODUCTION}

White-light cystoscopy (WLC) is the current standard for bladder cancer diagnosis and follow-up. 5-aminolaevulinic acid (5-ALA) or hexylaminolevulinate (HAL) are photoactive porphyrins that are preferentially taken up by dysplastic cells, which emit red fluorescence after exposure to blue light (blue-light cystoscopy, BLC) $(1,2)$. Blue light TURBT or biopsies has been shown to reduce the risk of early recurrence compared with WLTURBT improving the diagnostic accuracy of conventional cystoscopy for detecting bladder tumors and particularly carcinoma in situ (CIS) (1). Carcinoma in situ (CIS) of the urinary bladder is defined as a flat highgrade noninvasive transitional cell carcinoma (3). CIS is generally associated with a high risk of tumor progression. Bladder wash cytology should be positive in over $90 \%$ of patients with CIS, whereas conventional white light cystoscopy (WLC) may fail to detect CIS in up to $50 \%$ of cases. A full understanding of the presence and extent of CIS is crucial to treatment planning and followup, and may lead to improved treatment outcomes. From a quality of-life point of view it is also important to diagnose superficial flat lesions that will become evident in a few months, needing further transurethral resection. However, according to the 2011 European Association of Urology (EAU) guidelines, PDD cystoscopy should be restricted to those patients who are suspected of harboring a high-grade tumor, particularly CIS, and should not be used on a regular basis (1). To date, few studies have presented results of PDD cystoscopy compared with WLC in daily practice. The objective of the present study is to evaluate the diagnostic accuracy of HAL hydrochloride (Hexvix) PDD cystoscopy compared with standard WLC in daily practice.

\section{Materials ANd Methods}

61 consecutive patients with suspected or confirmed bladder cancer were recruited for the study from January 2008 until January 2015 (Table 1). Patients with suspected bladder cancer (positive cytology with negative WLC) or history of previous high-grade malignancy or CIS were included in the study. Exclusion criteria were gross hematuria, porphyria, known allergy to HAL, pregnancy or lactation, and intravesical Bacillus CalmetteGuerin (BCG) or chemotherapy within 3 months before HAL instillation. An observational, comparative, con- 
Table 1.

Characteristics of the sample.

\begin{tabular}{|lc|}
\hline Number of patients & 61 \\
\hline Male/female & $54 / 7$ \\
\hline Mean age & 77 \\
\hline Suspected CIS & 15 \\
\hline History of previous high-risk NMIBC & 46 \\
\hline Number of biopsies & 173 \\
\hline Positive biopsies to both BLC and WLC & 44 \\
\hline Positive biopsies only to BLC & 173 \\
\hline Positive biopsies only to WLC & 0 \\
\hline
\end{tabular}

trolled (within patient) study was carried out at our Center. All the data provided were collected as part of a routine clinical procedure. In addition, all patients gave their written informed consent to have their clinical records included in a dedicated database, and they were aware that their data, after having been made anonymous, would be used for clinical research purposes. The principles of the Declaration of Helsinki were followed.

HAL hydrochloride (Hexvix ${ }^{\circledR}$ ) in phosphate-buffered saline solution ( $50 \mathrm{ml}$ of $2.0 \mathrm{mg} / \mathrm{ml}(8 \mathrm{nM})$ solution) was instilled into the bladder via a standard catheter 1 hour before cystoscopy. The bladder was evacuated and inspected by white-light cystoscopy. A band filter on the lamp was then used to supply blue light (wavelength 380-450 $\mathrm{nm}$ ) for fluorescence cystoscopy. One dedicated and wellexperienced endourologist recorded the presence and number of positive lesions/suspicious areas compared with surrounding urothelium detected using white-light and blue-light cystoscopy. Biopsies/resection of each positive lesion/suspicious areas were always taken after the bladder was inspected under white and blue light. Random biopsies from normal-appearing urothelium were never taken. All procedures were performed under local or spinal anesthesia. The safety of HAL was evaluated by clinical examination at HAL instillation, during surgery, and in the postoperative period until discharge.

All papillary lesions/biopsies were collected and sent separately for histological analysis. Each biopsy was reviewed by a dedicated uropathologist blinded to detection method and patient history. All samples were evaluated and classified as normal urothelium, CIS, or urothelial neoplasia. Tumor stage was assigned according to the 2009 tumor- node-metastasis (TNM) classification (4). Grading of papillary lesions was assigned using the 1973 World Health Organization (WHO) classification (5).

Diagnoses of bladder tumor or CIS were considered as positive results, and the presence of normal urothelium in the biopsy specimen as negative result. Sensitivity, specificity and positive predictive value for each method were calculated. We compared the results per biopsy specimen for WLC and BLC using the McNemar test. Data was analyzed using SPSS for Windows version 17.0 (SPSS Inc., Chicago, IL, USA).

\section{RESULTS}

Analysis of WLC and BLC findings is presented in Table 2.
Table 2.

Results.

\begin{tabular}{|lc|}
\hline Positive biopsies & $129 / 173$ \\
\hline TaG1 & 17 \\
\hline TaG3 & 2 \\
\hline T1G3 & 21 \\
\hline CIS & 44 \\
\hline Sensibility of BLC for CIS & $100 \%$ \\
\hline Specificity WLC/BLC & $39 \%$ \\
\hline Predictive positive value BLC/WLC & $80 \% / 48.5 \%$ \\
\hline
\end{tabular}

61 BLC (54 men and 7 women; mean age 77 years) were performed. 15/61 (24.5\%) with suspected initial diagnosis of NMIBC (positive cytology and negative WLC) and $46 / 61(75.5 \%)$ with a history of high-risk NMIBC. We performed a total of 173 biopsies/TURBT of suspicious areas: 129 positive only to the BLC and 44 both positive to WLC and BLC.

84/173 biopsies/TURBT were positive for cancer with the following histology: 44 CIS, 17 TaG1, 2TaG3, 21 T1G3. All 84 NMIBC were positive to the BLC, while 35/84 of them were positive to the WLC with a sensitivity of BLC and WLC respectively of $100 \%$ and $41.7 \%$.

The 49 lesions detected only by the BLC had the following histology: 29 CIS, 14 TaG3, 6 T1G3.

Sensitivity of WLC for high-grade NMIBC and CIS was $34.1 \%$ and $39 \%$ respectively while sensitivity of BLC for high-grade NMIBC and CIS was 100\%.

The specificity of the WLC was $79.9 \%$ compared to $48.5 \%$ of the BLC. The positive predictive value of BLC and WLC were respectively 48\% (95\% CI 0.447-0.523) and $79 \%$ (95\% CI 0.856-0.734).

\section{Discussion}

As expected, our findings were consistent with the reports of previous studies that HAL was more sensitive for detection of CIS than WLC $(1,6-8)$. This is important as the data were consistent between studies performed in North America and Europe, where this technology has been approved by regulatory authorities in 27 countries. HAL also received US Food and Drug Administration approval in May, 2010.

The ability of HAL to detect both visible and occult CIS could provide a more accurate, site-directed approach to the identification of CIS.

BLC detected more CIS lesions than did WLC in patients with primary cancer. These data showed that the addition of BLC improved detection of CIS and might have resulted in a more complete determination of the extent of CIS. In addition, some patients who appeared normal by WLC were found to have CIS by BLC only. These data support an approach utilizing both WLC and BLC in order to optimize detection of CIS and determine the extent of CIS.

In the present study we evaluated the diagnostic accuracy of HAL PDD versus WLC for diagnosis of CIS and bladder tumors in daily practice. The sensitivity of BLC 
biopsies was significantly higher than sensitivity of WLC technique.

Fradet et al. compared Hexvix fluorescence cystoscopy with WLC for detecting CIS in a multicenter study on 298 patients (6). Overall, more CIS were found by Hexvix than by WLC: of a total of 113 CIS lesions diagnosed in 58 patients, 104 (92\%) were detected by HAL cystoscopy and 77 (68 \%) by WLC, thus leading to the conclusion that BLC can diagnose CIS that may be missed with WLC (6).

Similar conclusions were reached by Lerner et al. (7) in a recently published study using HAL in 551 patients. Presence of CIS is associated with higher risk of tumor progression and can significantly change the follow-up schedule and treatment algorithm toward BCG instillations instead of farmorubicin if associated with a papillary tumor confined to the mucosa (pTa) or toward cystectomy if its diagnosis is associated with recurrent highgrade papillary tumor invading the subepithelial connective tissue (pTl).

There are no data, however, regarding detection of CIS in extravesical sites using HAL. These sites can be a reservoir for CIS and explain, at least in part, the failure of BCG to completely clear the lower urinary tract of CIS in some patients.

This represents an important gap in our detection armamentarium that could possibly be addressed by further clinical trials of florescence cystoscopy or other novel imaging systems for detection of CIS in the upper urinary tract and prostatic urethra (9).

\section{REFERENCES}

1. Babjuk M, Oosterlinck W, Sylvester R, et al. EAU guidelines on nonmuscle-invasive urothelial carcinoma of the bladder, the 2011 update. Eur Urol. 2011; 59:997-1008.

2. Jocham D, Stepp H, Waidelich R. Photodynamic diagnosis in urology: state of the art. Eur Urol. 2008; 53:1138-1150.

3. Epstein JI, Amin MB, Reuter, VR, et al. The World Health Organization/International Society of Urological Pathology consensus classification of urothelial (transitional cell) neoplasms of the urinary bladder. Bladder Consensus Conference Committee. Am J Surg Pathol. 1998; 22:1435-48.

4. Sobin LH, Gospodarowicz MK, Wittekind C, eds TNM classification of malignant tumors (UICC International Union Against Cancer), 7th edn. Wiley-Blackwell, New York 2009, p. 262-5.

5. Mostofi FK, Sobin LH, Torloni H. Histopathological typing of urinary bladder tumours. In: International histological classification of tumours. World Health Organisation, Geneva 1973.

6. Fradet Y, Grossman HB, Gomella L, et al. A comparison of hexaminolevulinatefluorescence cystoscopy and white light cystoscopy for the detection of carcinoma in situ in patients with bladder cancer: a phase III, multicenter study. J Urol. 2007; 178:68-73.

7. Lerner SP, Liu H, Wu M-F, et al. Fluorescence and white light cystoscopy for detection of carcinoma in situ of the urinary bladder. Urol Oncol. 2012; 30:285-289.

8. Schmidbauer J, Witjes F, Schmeller N, et al. Improved detection of urothelial carcinoma in situ with hexaminolevulinate fluorescence cystoscopy. J Urol. 2004; 171:135-138.

9. Goh AC, Lerner SP. Application of new technology in bladder cancer diagnosis and treatment. World J Urol. 2009; 27:301-7.

\author{
Correspondence \\ Vito Lacetera, MD, Urologist \\ vlacetera@gmail.com \\ Ubaldo Cantoro, MD, Urologist (Corresponding Author) \\ ubaldocantoro@tiscali.it \\ Lorenzo Montesi, MD, Resident in Urology \\ lorenzomontesi@yahoo.it \\ Bernardo Cervelli, MD, Urologist \\ bernardino.cervelli@ospedalimarchenord.it \\ Antonio Cicetti, MD, Urologist \\ antonio.cicetti@ospedalimarchenord.it \\ Giuliana Gabrielloni, MD, Urologist \\ giuliana.gabrielloni@ospedalimarchenord.it \\ Domenico Milella, MD, Urologist \\ domenico.milella@ospedalimarchenord.it \\ Michele Montesi, MD, Urologist \\ michele.montesi@ospedalimarchenord.it \\ Roberto Morcellini, MD, Urologist \\ roberto.morcellini@ospedalimarchenord.it \\ Gianni Parri, MD, Urologist \\ gianni.parri@ospedalimarchenord.it \\ Emilio Recanatini, MD, Urologist \\ emilio.recanatini@ospedalimarchenord.it \\ Valerio Beatrici, MD, Urologist \\ beatrici@libero.it \\ Azienda Ospedaliera Ospedali Riuniti Marche Nord \\ Piazzale Cinelli 4 - 61121 Pesaro, Italy \\ Daniele Cantoro, MD, Urologist \\ danidoc2580@alice.it \\ Ospedale di Ascoli Piceno, Ascoli Piceno, Italy
}

